\title{
D’Homère à la rhétorique : un certain art du mensonge
}

Von Homer zur Rhetorik. Eine gewisse Kunst des Lügens

From Homer to rhetoric: a certain art of lying

\section{Alain Malissard}

\section{(2) OpenEdition}

Journals

Édition électronique

URL : http://journals.openedition.org/ceg/1673

DOI : $10.4000 /$ ceg. 1673

ISSN : 2605-8359

Éditeur

Presses Universitaires de Provence

Édition imprimée

Date de publication : 1 décembre 2014

Pagination : 63-74

ISSN : 0751-4239

Référence électronique

Alain Malissard, " D'Homère à la rhétorique : un certain art du mensonge », Cahiers d'Études

Germaniques [En ligne], 67 | 2014, mis en ligne le 17 décembre 2017, consulté le 04 novembre 2020

URL : http://journals.openedition.org/ceg/1673; DOI : https://doi.org/10.4000/ceg.1673 


\title{
D'Homère à la rhétorique : un certain art du mensonge
}

\author{
Alain MALISSARD \\ Université d'Orléans
}

Des origines de notre littérature à nos jours, en passant par les temps d'invention d'un art ou d'une technique du discours, la parole et les mots qui peuvent persuader l'auditoire ont le plus souvent suscité la méfiance. Le mensonge peut en effet toujours y prendre place. Cette méfiance est explicable, et souvent justifiée.

Dans les textes fondateurs de la littérature occidentale, on trouve en effet toute une série de mensonges et même la condamnation d'un certain usage de la vérité.

\section{Au temps de la guerre de Troie}

\section{Une série de mensonges}

Le sacrifice d'Iphigénie

Dès le début de l'expédition des Grecs contre Troie, la flotte rassemblée par Agamemnon dans le port d'Aulis attend en vain que le vent se lève. Consulté, le devin Calchas révèle qu'une offense a été faite à Artémis et que la déesse exige qu'Iphigénie, fille d'Agamemnon, lui soit sacrifiée. Le roi doit alors inventer un prétexte : il demande par lettre à son épouse Clytemnestre de venir avec sa fille et lui explique qu'Achille refuse de partir pour Troie, s'il n'épouse pas d'abord Iphigénie. C'est donc un mensonge qui rend possible le départ de la flotte. Ce ne sera pas le seul : le triomphe final des Grecs n'est rendu possible que par une série complexe de mensonges, dont nous ne retiendrons que deux exemples. 


\section{Philoctète et Néoptolème}

Avant de monter sur le bûcher ${ }^{1}$, Héraclès a donné à son ami Philoctète son arc et ses flèches qui atteignent toujours leur cible. Muni de ces armes efficaces, Philoctète part d'Aulis avec Agamemnon, mais il est, au cours d'une escale, mordu au pied par un serpent; jointe à ses plaintes et à ses lamentations, sa blessure devient si malodorante et si effroyable sur le navire qui le transporte qu'Ulysse le fait débarquer dans l'île de Lemnos où il le laisse seul et abandonné.

Des années de siège se passent sans que les Grecs obtiennent un résultat, Achille est mort, et le devin troyen Hélénos, fait prisonnier par Ulysse, confirme ce qu'avait dit déjà l'oracle de Delphes : Troie ne peut être prise sans les armes d'Héraclès. Leur récupération devient donc absolument nécessaire et Ulysse part pour Lemnos en compagnie du jeune Néoptolème, qui est le fils d'Achille.

Dès qu'ils débarquent ${ }^{2}$, Ulysse explique à Néoptolème que c'est lui qui doit s'approcher de Philoctète et récupérer l'arc ; pour gagner la confiance du malade solitaire, il doit lui dire qu'après la mort d'Achille les Grecs l'ont fait venir à Troie, mais qu'ils ont refusé de lui donner les armes de son père, parce qu'Ulysse les avait d'abord exigées ; ce mensonge fera de lui un ennemi d'Ulysse, mettra Philoctète en confiance et il pourra se saisir des armes qui assureront la défaite des Troyens.

A cette étape importante du récit, il s'agit à présent de faire mentir quelqu'un. Dans un premier temps cependant, Néoptolème refuse : "Que m'enjoins-tu donc là, si ce n'est pas mentir ${ }^{3}$ ? », demande-t-il. Dans la pièce de Sophocle, Néoptolème se laissera finalement convaincre, mais sera continuellement en lutte avec lui-même, car "ces vilains artifices ${ }^{4}$ » lui font horreur.

\section{Le cheval de Troie}

L'histoire est trop connue pour y revenir. Qu'il suffise donc de dire que le cheval est en lui-même un objet trompeur. Il est le fruit de l'intelligence madrée d'Ulysse ; c'est une ruse de guerre, un mensonge-objet. En tant que piège, il ne pourra cependant fonctionner qu'au prix d'un étouffement de la vérité, ou qu'avec l'appui d'un mensonge exprimé par la parole. Le mensonge

\footnotetext{
${ }^{1}$ Jalouse d'Iole, Déjanire, épouse d'Héraclès, lui a donné une robe empoisonnée par le sang du Centaure Nessus. La robe s'attache aux chairs d'Héraclès et lui cause d'atroces souffrances ; pour y mettre fin, Héraclès monte sur un bûcher et décide Philoctète, ou son père, à y mettre le feu.

${ }^{2}$ Eschyle et Euripide avaient traité le sujet. Nous nous référons ici à la pièce de Sophocle qui seule a subsisté.

${ }^{3}$ Sophocle, Philoctète, trad. A. Dain, P. Mazon et J. Irigoin, Paris, Les Belles Lettres (Collection des Universités de France), 4 éd. revue 1990, v. 100.

${ }^{4}$ Ibid., v. 88
} 
sera celui de Sinon, sur lequel nous allons revenir en détail. Il nous faut en effet examiner d'abord quel sort les récits d'Homère réservent à la vérité.

\section{Les malheurs de la vérité}

\section{À Troie}

Quand l'énigmatique idole se dresse devant Troie, Laocoon, prêtre de Poséidon, vient dire aux Troyens qu'il s'agit d'un piège ; pour le prouver, il frappe les flancs du cheval qui sonnent creux, mais les Troyens l'accusent de sacrilège et tiennent d'autant moins compte de ses avertissements que d'affreux serpents surgissent soudainement de la mer et viennent l'étouffer avec ses trois enfants.

Rappelons aussi, sans qu'elle ait de rapport direct avec l'épisode du cheval, la présence à Troie de Cassandre, qu'Apollon, à qui elle s'est refusée, a condamnée à toujours dire la vérité sans être jamais crue.

À Troie, en fait, la vérité est, soit étouffée, soit inaudible.

\section{Ulysse}

Plus probant encore est le cas d'Ulysse. L'homme aux mille tours, le rusé, le menteur, le héros de la mètis ${ }^{5}$ grecque se laisse en effet prendre au piège de la vérité et contribue dans ce cas sans le vouloir à l'apologie du mensonge que portent en elles l'Iliade et l'Odyssée.

Après avoir aveuglé le Cyclope et fait sortir de la grotte ceux de ses compagnons qui ont survécu, Ulysse s'éloigne de la terre à force de rames. Avec l'astuce qui le caractérise, il a d'abord dit au Cyclope qu'il s'appelait «Personne »; maintenant qu'il est au large et à l'abri des énormes rochers que les Cyclopes ameutés jettent dans sa direction, il éprouve dans la joie de son succès le besoin de clamer son véritable nom : "Cyclope, si quelqu'un des mortels te demande jamais qui t'infligea la honte de te priver de l'œil, dislui que c'est Ulysse saccageur de cités qui te rendit aveugle, Ulysse, fils de Laërte, qui réside en Ithaque ${ }^{6} »$.

Mal lui en prend, car le Cyclope est le fils de Poséidon, auquel il s'adresse en demandant vengeance, et le dieu des mers ne manquera jamais de déclencher sur le Grec des tempêtes dévastatrices!

Dans les textes fondateurs de notre littérature et dans ceux qui en dérivent, c'est-à-dire au cœur même de la légende, on trouve donc à la fois une apologie du mensonge et une critique de la vérité.

Le mensonge y est toujours justifié par l'intérêt collectif (c'est le cas d'Iphigénie et de Néoptolème) ou par les lois de la guerre (c'est le cas du

\footnotetext{
${ }^{5}$ " Puissance de réflexion », pris au sens de « ruse », « artifice », voire de « perfidie ».

${ }^{6}$ Homère, Odyssée, trad. nouv. de Mario Meunier, Paris, Albin Michel (Cercle du bibliophile), 1967, 9, v. 502-506.
} 
cheval de Troie) et il est en outre efficace : la flotte d'Agamemnon peut partir, l'arc et les flèches d'Héraclès sont récupérés, Troie tombe aux mains des Grecs.

À cette apologie du mensonge s'ajoute une condamnation de la vérité. Dans l'épisode du cheval, inaudible ou étouffée, elle est imperceptible à un peuple aveuglé par les passions ; quand les Troyens, par exemple, déplacent le cheval, ils entendent, à quatre reprises, les armes cliqueter à l'intérieur, mais n'y prêtent aucune attention. Chez Ulysse, elle marque le passage de la mètis à l'ubris ${ }^{7}$ et apparaît nettement comme une manifestation d'orgueil et d'excès ; à l'inverse du mensonge, elle est irréfléchie, injustifiée, inefficace et même néfaste puisqu'elle engendre une série de catastrophes.

De cette apologie du mensonge et de cette méfiance à l'égard de la vérité, la rhétorique se souviendra.

\section{La persuasion par le mensonge}

Pour analyser le fonctionnement du mensonge, examinons ses raisons dans la tragédie de Sophocle et ses méthodes dans l'épopée de Virgile.

\section{Les raisons du mensonge : Néoptolème}

On vient de voir que, dans un premier mouvement, Néoptolème rejette la méthode proposée par Ulysse. Pour ne pas recourir au mensonge, il propose deux solutions, soit utiliser la force ${ }^{8}$, soit user de persuasion ; Ulysse lui répond que l'emploi de la force est impossible, car l'arc et les flèches qui se trouvent entre les mains de Philoctète sont extrêmement dangereux ; en vieux routier rusé, il considère, « expérience faite », que « ce qui mène tout, c'est la langue et non les actes ${ }^{9} »$.

Pour Néoptolème cependant, on peut se servir de la langue et des discours sans mentir. User de la parole et des mots, c'est aussi rappeler l'enjeu à Philoctète, faire appel à la raison, expliquer, convaincre ou persuader, autrement dit partir de la vérité et rester dans le juste et le vrai pour atteindre l'objectif. Mais, aux yeux d'Ulysse, la haine de Philoctète pour les Grecs est trop violente ; pour déjouer sa fureur, il faut mentir et captiver son esprit ; Néoptolème doit «par [son] langage tromper l'âme de Philoctète ${ }^{10}$ ». Seul le mensonge sera efficace : il obtiendra un résultat et engendrera en outre un

\footnotetext{
${ }^{7}$ « Démesure».

${ }^{8}$ Le recours à la force est souvent opposé à la persuasion. Dans le Gorgias, c'est, par exemple, ce que propose Calliclès : PlATON, Gorgias, texte établi et traduit par Alfred Croiset (avec la collaboration de Louis Bodin), Paris, Les Belles Lettres (Collection des Universités de France), 1949, 482c-486d.

${ }^{9}$ SOPHOCLE, Philoctète, v. 99.

${ }^{10}$ Ibid., v. 54-55
} 
double profit : Néoptolème récupérera l'arc et gagnera la réputation de brave et d'adroit.

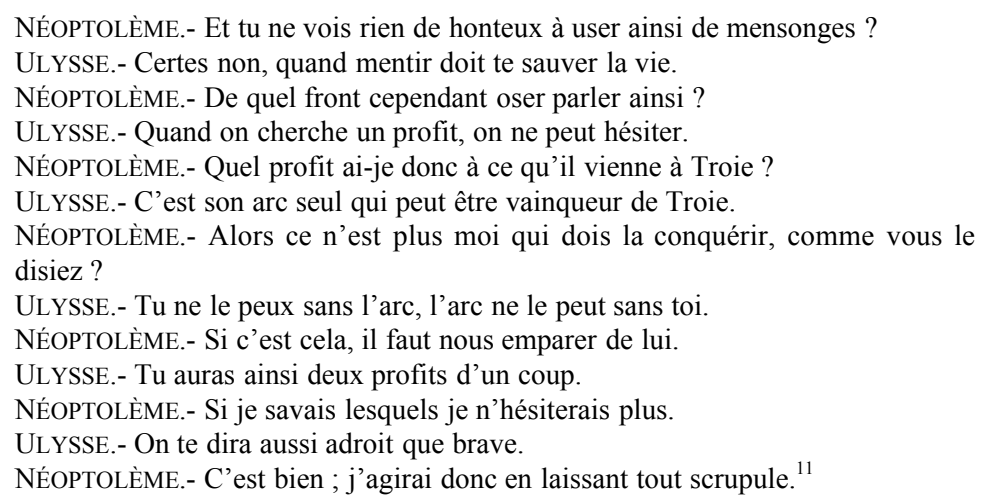

Séduit par les arguments d'Ulysse, Néoptolème accepte le principe du mensonge et saura même se montrer très éloquent !

Cette éloquence est plus sensible encore dans le discours que Virgile place dans la bouche de Sinon au chant deux de l'Énéide ${ }^{12}$.

\section{Les méthodes du mensonge : le discours de Sinon}

Ayant décidé de prendre Troie par la ruse, les Grecs ont fait semblant de renoncer au siège et ont laissé devant les murs de Troie un gigantesque cheval de bois dont les flancs cachent un commando de guerriers commandés par Ulysse, l'auteur du projet. Tout à leur joie, les Troyens sortent de la ville, se rassemblent autour du cheval et se demandent ce qu'ils doivent faire : faut-il le jeter à la mer, le brûler, en percer les flancs ou l'installer dans la ville en signe de victoire ? Comme on l'a vu, le cheval est un mensonge-objet qui a besoin du discours pour fonctionner. Virgile a donc créé un personnage, Sinon, un Grec plein de charme qui se fait passer pour une victime d'Ulysse et va persuader les Troyens de faire entrer le cheval dans la ville en abattant une partie de leurs remparts; des bergers, auxquels il vient de se livrer spontanément le traînent aux pieds de Priam et Virgile place son discours dans le long récit qu'Énée fait à Didon de ses malheurs et de la chute de Troie.

Il y a en fait deux prises de parole de Sinon, la seconde étant beaucoup plus courte que la première. Elles suivent, l'une et l'autre, une organisation générale du discours, dont le plan a été tracé très tôt $\mathrm{t}^{13}$; on y trouve donc un

\footnotetext{
${ }^{11}$ Ibid., v. 108-120.

${ }^{12}$ Virgile, Énéide, texte établi par Henri Goelzer et René Durand et traduit par André Bellessort, Paris, Les Belles Lettres (Collection des Universités de France), 1970, 2, 57-198.

${ }^{13}$ Voir Françoise DESBORDES, La rhétorique antique, Paris, Hachette, 1996, p. 80-84.
} 
exorde, une captatio benevolentiae, une narration, une argumentation, une péroraison.

\section{Premier discours (vers 69 à 144)}

Captatio benevolentiae (69-80). Elle consiste pour Sinon à capter par ses plaintes l'attention des Troyens rassemblés autour du cheval. Dès qu'on l'écoute, il se calme et, parlant de lui-même à la troisième personne, il se met en quelque sorte à la place de ses auditeurs : « Si la Fortune a fait de Sinon un malheureux, elle ne fera pas de lui, dans son acharnement, un menteur et un fourbe ${ }^{14} »$.

Dans l'esprit des Troyens, encore indécis et soupçonneux, Sinon glisse ainsi l'idée que sa situation est telle qu'il ne peut dire que la vérité. Commence alors la narration de ses malheurs.

Première narration (8-100). Le récit, assez bref, mais pathétique, présente Sinon comme une victime du ressentiment d'Ulysse.

Interruption (100-104). Avec une très grande habileté rhétorique, Sinon interrompt brutalement son récit et déclare soudainement que les Troyens ne sont pas obligés de l'écouter; puisqu'il est Grec, ils peuvent le tuer tout de suite : " voilà ce que voudrait l'homme d'Ithaque et que les Atrides paieraient cher ${ }^{15}$ ", déclare-t-il. À ce moment de la narration les Troyens sont piégés : le tuer serait ne jamais avoir la fin du récit, mais ce serait surtout faire ce que voulaient précisément faire les Grecs ; ils se trouvent donc en quelque sorte contraints d'écouter la suite du discours et déjà d'y ajouter foi.

Reprise de la narration (105-136). Dans cette seconde partie de la narration, Sinon révèle que les Grecs ont depuis longtemps le désir de lever le siège et de rentrer chez eux. Pour que la mer leur soit favorable, l'oracle de Delphes leur a demandé de sacrifier un des leurs à Poséidon et c'est lui, Sinon, que, sur les conseils d'Ulysse, Calchas a fini par désigner! Aux Grecs, il faut ainsi Iphigénie pour partir et Sinon pour revenir! Mais lui s'est caché et c'est pourquoi il se trouve maintenant, misérable, devant eux.

Péroraison (137-144). Dans le style pitoyable et grandiloquent qui est caractéristique des péroraisons, Sinon évoque sa patrie, ses enfants, son père, sa propre situation et fait appel à la pitié des assistants ; il ne leur donne cependant aucun conseil. Il faut en effet que leur confiance naisse de l'émotion qu'il fait ainsi surgir en eux. Comme dans tout discours conduit selon les règles, la fin rejoint le début: Sinon n'est pas un menteur; il exprime une vérité dont les Dieux peuvent se montrer garants, et c'est eux qu'il invoque à nouveau quand il implore la pitié de Priam : " par les dieux d'En Haut, par les Puissances divines qui savent la vérité, par ce qu'il y a encore chez les mortels de justice inviolée, je t'en supplie, aie pitié de si grandes épreuves, aie pitié d'un cœur qui ne les méritait pas ${ }^{16}$ ! »

\footnotetext{
${ }^{14}$ VIRGILE, Énéide, v. 79-80.

${ }^{15}$ Ibid., v. 104.

${ }^{16}$ Ibid., v. 140-144.
} 


\section{Deuxième discours (vers 154 à 194)}

Après la brève intervention de Priam (145-153), les Troyens sont persuadés que Sinon dit vrai ; il peut donc, dans un second discours, moins long, mais décisif, parvenir à son objectif final qui est d'amener les Troyens à percer leurs remparts pour faire entrer le cheval dans la ville.

L'exorde (154-161) excite la curiosité : Sinon va produire au grand jour ce qui devait rester caché, mais, en échange des vérités qu'il va rapporter, il demande à Troie de rester fidèle à ses promesses : "Toi seulement, ville de Troie, sois fidèle à tes promesses et, gardée par moi, garde moi ta parole si je te dis la vérité ${ }^{17}$. »

La narration (162-184) explique les raisons pour lesquelles les Grecs ont placé le cheval devant Troie : c'est une offrande que les Grecs font à Pallas en expiation du vol du Palladium ${ }^{18}$.

L'argumentation finale (185-194) révèle que Calchas a voulu que le cheval soit gigantesque, afin qu'il ne puisse pas entrer dans Troie. Les Troyens ne doivent pas faire ce que voulaient les Grecs : ils doivent donc faire entrer le cheval dans la ville.

Ainsi se trouve réalisé ce qui était l'objet même d'un long discours : la confiance a été établie, les Troyens prennent pour vrai ce qui est faux et passent à l'action. Une fois de plus, le mensonge est efficace.

\section{La conscience du mensonge}

Dans les deux exemples que nous avons cités apparaît cependant une sorte de critique du mensonge qui s'exprime sous deux formes : le remords et l'intervention du narrateur.

Dans la tragédie de Sophocle d'abord, Néoptolème se reproche d'avoir menti. Il finira par dire la vérité à Philoctète et essaiera de le convaincre. Toute la tragédie se présente ainsi comme une réflexion sur le mensonge, et on a pu dire que le conflit tragique n'est pas entre deux individus, mais dans le cœur ou l'âme d'un seul ${ }^{19}$. Une fois de plus, cependant, la vérité n'est pas efficace : Philoctète s'obstine dans son refus et l'aveu de Néoptolème conduit à une aporie. Pour respecter la légende et la tradition, Sophocle doit donc recourir à un deus ex machina et faire intervenir Héraclès, qui ordonne à Philoctète de partir avec Ulysse et Néoptolème pour participer au siège de Troie.

Chez Virgile, le discours de Sinon est contenu dans le discours d'Énée qui peut l'interrompre et le commenter pour signaler le mensonge ; les mots 《 traitre », « scélérat », « menteur », « ruse » et « artifice » viennent ainsi

\footnotetext{
${ }^{17}$ Ibid., v. 160-161.

${ }^{18}$ Le Palladium (ou Palladion) est une statue de Pallas-Athéna dont, au cours d'une expédition sanglante au cœur de Troie, Diomède et Ulysse s'étaient emparés pour la transporter dans le camp des Grecs.

${ }^{19}$ SOPHOCLE, Philoctète, notice p. 4-5.
} 
marquer les étapes d'un discours dont ils démontent les artifices et les méthodes en même temps qu'ils les reproduisent.

Le mensonge est donc dénoncé, mais il a été efficace, puisque les Troyens sont aveuglés à tel point qu'ils n'entendent pas le cliquetis des armes à l'intérieur du cheval quand il bute sur un obstacle au cours de son déplacement vers l'intérieur de Troie.

D'un point de vue moral, Sophocle et Virgile condamnent tous deux le mensonge, mais ils savent, le premier, parfaitement le justifier, le deuxième, parfaitement le faire fonctionner. Ils en connaissent et en reconnaissent l'efficacité et savent aussi que le mensonge réussi devient la vérité de celui qui l'écoute et le croit ; ils utilisent, l'un et l'autre, l'ambiguité de la parole.

C'est que Sophocle écrit Philoctète en 409 et Virgile l'Énéide à la fin du premier siècle avant J.-C. Ils ont donc été formés, l'un et l'autre, à la rhétorique et à la sophistique qui leur ont montré que toute parole, tout discours, tout logos, est double. Les deux exemples que nous avons choisis nous engagent donc à poser la question de l'usage que la rhétorique peut faire du vrai et du faux.

\section{La rhétorique antique : mensonge et liberté}

La rhétorique et les Sophistes, qui peuvent être confondus sur le plan politique, sont nés en Sicile grecque au moment de la chute des Tyrans, c'està-dire en même temps que la démocratie. La démocratie rend en effet nécessaire de convaincre le peuple, assemblé pour décider des lois, ou les juges qui doivent dire le droit, les deux n'ayant rapidement fait qu'un à Athènes, puisque c'est le peuple qui exerce la justice ${ }^{20}$.

L'apparition de la rhétorique marque en fait le passage d'une parole sacralisée à une parole-dialogue ${ }^{21}$. La parole sacralisée du chef, du prêtre, du devin, voire du Dieu lui-même, était détentrice d'une vérité efficace (alètheia) qu'on ne pouvait oublier $(\text { a-lèthè })^{22}$; la parole-dialogue propose au contraire une vérité marquée d'ambiguïté ${ }^{23}$. La parole sacralisée du prêtre baignait en effet dans la lumière de l'évidence; elle tirait son efficacité de la confiance (pistis) qu'elle engendrait naturellement ; la parole-dialogue au contraire recèle une part d'ombre ; elle ne produit pas spontanément la confiance, mais cherche à l'obtenir : elle doit convaincre. On passe ainsi de la pistis, qui est confiance en la parole, à la peitho qui est charme et séduction par la parole (timbre de la voix, choix des mots, phrasé, etc.). Or, pour

\footnotetext{
${ }^{20}$ Voir DESBORDES, Rhétorique, p. 14-16.

${ }^{21}$ Marcel DeTiEnNe, Les Maîtres de Vérité dans la Grèce archaïque, Paris, Le Livre de Poche (collection Références), 2006 [1 $1^{\mathrm{re}}$ éd. Maspéro, 1967], p. 178-183.

${ }^{22}$ Ibid., p. 79-82.

${ }^{23}$ Ibid., p. $144-151$ et 203-209.
} 
convaincre, la peitho peut, soit soutenir l'alètheia, soit user de l'apatè (tromperie) $^{24}$.

Avec la rhétorique, aussitôt définie comme un art de convaincre par la parole, le logos se fait autonome et forge ses propres lois; la parole devient un instrument dont des procédés divers et des techniques (technè) vont favoriser l'utilisation. Le rhéteur et le sophiste sont des techniciens du $\log 0 s^{25}$.

Sans aller plus loin dans le détail, trois questions peuvent alors se poser : celle de l'ambiguïté du discours, celle de l'utilisation du mensonge et celle de la liberté qu'apporte la parole-dialogue quand on la confronte à la parole sacralisée.

\section{Ambiguïté et identité}

Dans le cadre de la démocratie, qu'il s'agisse d'adopter une loi ou de rendre un jugement, il y aura nécessairement devant le peuple un pour et un contre. Le juge, par exemple, devra entendre la défense et l'accusation du prévenu, mais, pour qu'il puisse appliquer la loi, il faudra qu'il distingue le vrai du faux, la parole vraie de la parole fausse. Or rien ne distingue un discours vrai d'un discours faux ; ils sont totalement et parfaitement identiques $^{26}$. En participant à un colloque, je déclare que je suis heureux d'être devant mes auditeurs, mais si je mens, je prononce les mêmes mots. À chacun de juger !

Dans le cas du peuple-juge, la décision d'acquitter ou de condamner ne peut donc être prise qu'en conséquence de l'effet produit sur l'auditeur par un discours qui peut, soit contenir la vérité, soit dissimuler le mensonge, puisqu'il n'existe dans l'Antiquité, ni expert, ni véritable possibilité d'investigation.

L'opinion, puis la décision du juge ne sont alors commandées ou déterminées que par la force et la qualité du discours qu'on lui adresse et l'orateur habile peut aussi bien convaincre du vrai que du faux. Le juge devient son esclave ; il dépend du choix et de la liberté de l'orateur. C'est ce que dit Gorgias :

SOCRATE.- [...] Fais-nous connaître quelle est cette chose que tu dis être pour l'homme le plus grand des biens, et que tu fais profession de produire.

GORGIAS.- C'est celle qui est réellement le bien suprême, celle qui donne à qui la possède la liberté pour lui-même et la domination sur les autres dans sa patrie. SOCRATE.- Mais enfin qu'entends-tu par là ?

GORGIAS.- J'entends le pouvoir de persuader par le discours les juges au tribunal, les sénateurs au Conseil, le peuple dans l'Assemblée du peuple et de même dans toute autre réunion qui soit une réunion de citoyens... ${ }^{27}$

\footnotetext{
${ }^{24}$ Ibid., p. 125-133 et DESBORDES, Rhétorique, p. 10.

${ }^{25}$ DETIENNE, Les Maîtres de Vérité, p. 181-182 et 206 ; DESBORDES, Rhétorique, p. 31-37.

${ }^{26}$ DESBORDES, Rhétorique, p. 17-18 et 173-174.

${ }^{27}$ Platon, Gorgias, 452 d-e.
} 
À partir du moment où Platon s'est attaqué aux Sophistes tels que Gorgias ou Protagoras, cette duplicité du logos, cette identité dans l'expression de la vérité et du mensonge, a été chez les anciens Grecs, puis Romains, une source continuelle de débats moraux. On a essentiellement accusé la rhétorique d'être un art de persuader par le mensonge.

\section{Un art du mensonge?}

La question ne peut que se poser car la tentation est forte, devant une assemblée ou un juge, de mentir pour faire valoir son point de vue ou pour sauver son client. Les Sophistes grecs et les logographes, dont le métier était d'écrire des discours, estimaient d'ailleurs qu'on peut par la parole faire exister ce qui n'est pas.

Le fait est qu'à Rome, après cinq siècles d'existence et de perfectionnement de la rhétorique, l'usage du mensonge et l'indifférence à la vérité sont devenus monnaie courante. Dans De Oratore de Cicéron, le grand orateur Antoine, qui s'apprête à exposer sa conception de l'éloquence, déclare ainsi, d'une manière moins cynique que pragmatique :

[...] [N]ous-mêmes il nous arrive de soutenir alternativement des causes toutes contraires. Ainsi, non seulement Crassus parlera contre moi, ou moi contre Crassus, quoique l'un de nous deux doive nécessairement avoir tort; mais quelquefois même l'un de nous deux, après avoir soutenu un parti dans une cause, soutiendra le parti contraire dans une cause pareille; et cependant la vérité est toujours une. J'ai donc à vous entretenir d'une chose qui est appuyée sur le mensonge, qui conduit rarement à la vérité $[\ldots]^{28}$

Dans le même ouvrage, Cicéron lui-même, qui se fait pourtant de la rhétorique une idée plus haute et plus noble, reconnaît cependant, à propos des portraits à charge qu'on peut faire et de la raillerie : «qu'il ne faut pas se faire scrupule d'enchérir un peu sur la vérité en recourant à de petits mensonges (mendaciunculis) ${ }^{29} »$. Ailleurs, il défend le droit qu'a un orateur de se contredire en rappelant que l'orateur ne parle pas selon sa conscience, mais selon la cause qu'il défend, et Marcus Antonius ne donnait jamais le texte écrit de ses discours afin de pouvoir au besoin les désavouer ${ }^{30}$. Quintilien enfin, qui soutient pourtant que « là où la cause est injuste, il n'y a pas de rhétorique ${ }^{31} »$, ajoute quelques lignes plus bas : «bien que l'orateur $2,7,30$

${ }^{28}$ CiCÉrOn, De l'orateur, traduit sous la direction de M. Nisard, Paris, Dubochet, 1840, t. 2,

${ }^{29}$ Ibid., 2, 59, 241.

${ }^{30}$ CiCÉron, Pour Cluentius, traduit sous la direction de M. Nisard, Paris, Dubochet, 1840, t. 2, 50, 140 .

${ }^{31}$ QuinTILIEN, Institution oratoire, éd. et tr. par Jean Cousin, $3^{\mathrm{e}}$ tirage revu et corrigé par Guy Achard, Paris, Les Belles Lettres (Collection des Universités de France), 1976, t. 2, 2, 17, 31. 
doive très souvent s'appliquer à défendre la vérité, il n'en est pas toujours ainsi : l'intérêt public exige parfois qu'il soutienne aussi le faux ${ }^{32}$."

C'est qu'à Rome, et sans doute à Athènes aussi, les rhéteurs et les écoles de rhétorique n'enseignaient pas à parler selon sa conscience et à défendre ce qui était juste et vrai. Privilégiant toujours le résultat à obtenir et l'efficacité du discours, ils ne reculaient pas devant l'utilisation du mensonge persuasif et déconseillaient souvent l'usage d'une vérité qui pouvait être autodestructrice. Triompher, par exemple, dans une cause honteuse ou douteuse, donc difficile, était un moyen presque recommandé de se faire un nom et d'assurer sa notoriété.

$\mathrm{Au}$ temps des Sophistes grecs, comme au temps des avocats et des hommes politiques romains, la rhétorique a donc toujours pu être justement accusée de ne pas se préoccuper prioritairement de justice et de vérité, et de trop souvent mettre le talent au service du mensonge.

\section{Une marque de la liberté?}

Pour sa défense, l'art oratoire pourrait répondre qu'il laisse le choix entre la vérité et le mensonge, parce qu'il est une expression de la liberté. La rhétorique est en effet une production et une conséquence de la démocratie; née à Athènes en 510 av. J.-C., après la chute du tyran Hippias, fils de Pisistrate, qui marque le début du processus de démocratisation ${ }^{33}$, elle prospère à Rome avec la République ; à Athènes, elle disparaît pendant le bref épisode des Trente tyrans (404-403 av. J.-C.) ; à Rome, elle s'étiole sous l'Empire.

La critique des Sophistes que contient le Gorgias de Platon recèle déjà une critique implicite de la démocratie, et l'avocat historien Tacite, qui écrit sous le règne de l'empereur Trajan (81 ap. J.-C.) peut faire dire à Maternus, dans le Dialogue des orateurs :

[...] Cette grande et glorieuse éloquence d'autrefois est la fille de la licence, que des sots vont appelant liberté, la compagne des séditions, l'aiguillon d'un peuple sans frein ; ne connaissant pas l'obéissance ni le sérieux, opiniâtre, téméraire, arrogante, elle ne naît pas dans les États doués d'une sage constitution ${ }^{34}$.

C'est que, si la démocratie a en quelque sorte transformé la parole sacralisée en parole-dialogue, la rhétorique a transformé la parole-dialogue en une parole contradictoire nécessaire au débat et à la réflexion sur le juste et l'injuste, et c'est dans cette possibilité de contradiction, offerte par la liberté, que se glissent la tentation et la possibilité du mensonge.

\footnotetext{
${ }^{32}$ Ibid., 36.

${ }^{33}$ DESBORDES, Rhétorique, p. 11 et p. 14-15.

${ }^{34}$ TACITE, Dialogue des Orateurs, texte établi par Henri Goelzer et traduit par Henri Bornecque, Paris, Les Belles Lettres (Collection des Universités de France), 1967, 40, 2.
} 
Supprimer cette possibilité de contradiction et ne pas assumer le risque du mensonge, c'est soit rêver d'une parole-dialogue qui n'unirait que des complémentaires, soit accepter de revenir à la parole sacralisée qui deviendrait en fait la parole d'un seul, légitimée par le peuple. C'est d'ailleurs ce que dit Maternus quand il ajoute :

[...] Les honneurs accordés aux orateurs sont moins grands et leur gloire moins éclatante quand les mœurs des citoyens sont bonnes et qu'ils sont disposés à écouter le chef. À quoi bon développer son avis au Sénat, puisque l'élite des citoyens y tombe vite d'accord ? À quoi bon accumuler les discours devant le peuple, puisque, sur les intérêts publics, ce ne sont pas des incompétents et la foule qui délibèrent, mais le plus sage des hommes tout seul ? $^{35}$

Commencé à l'époque des Sophistes et de Platon, le débat dure encore et nous ne pouvons ici que poser le problème. Disons seulement pour conclure que c'est l'honneur d'une démocratie de laisser le champ libre aux débats et méfions-nous d'une condamnation sans nuance des risques du mensonge qui pourrait conduire à confisquer la parole au profit, en effet, d'un seul, réputé si sage ou reconnu si populaire qu'il serait considéré comme l'unique détenteur de la vérité.

Le mensonge est le risque de la liberté.

${ }^{35}$ Ibid., 41, 4. 